\title{
Gas Absorption and Pore Breathing of Metal-Organic Frameworks Studied Using in situ Environmental Transmission Electron Microscopy (ETEM)
}

Lucas R. Parent ${ }^{1,2}$, C. Huy Pham ${ }^{2}$, Joseph P. Patterson ${ }^{2,3}$, Michael S. Denny ${ }^{2}$, Seth M. Cohen ${ }^{2}$, Francesco Paesani $^{2}$, and Nathan C. Gianneschi ${ }^{1,2}$

1. Department of Chemistry, Department of Materials Science and Engineering, and Department of Biomedical Engineering, Northwestern University, Evanston, USA.

2. Department of Chemistry and Biochemistry, University of California, San Diego, San Diego, USA.

${ }^{3 .}$ Laboratory of Materials and Interface Chemistry and Center of Multiscale Electron Microscopy, Department of Chemical Engineering, Eindhoven University of Technology, Eindhoven, NL.

Metal-organic frameworks (MOFs), a class of periodic mesoporous nanomaterials, exhibit promising small molecule adsorption properties for gas storage/separation, comparable to those of zeolites [1]. However, little is currently known about the fundamental mechanisms involved in functional gas absorption/release ("pore breathing") behavior of these nanostructures, which limits our ability to controllably design and bulk-produce MOFs that are optimized for specific gas storage or separation applications. The bulk gas-storage capacity and kinetics of uptake/release of porous materials, including MOFs, are routinely screened using bulk gas adsorption analysis, such as breakthrough analysis and dynamic loading, supported by (bulk) powder X-ray diffraction (PXRD) [2,3]. However, these techniques cannot reveal the underlying molecular-scale mechanisms that give rise to the observed bulk gas storage properties. At the fundamental level, gas adsorption(/release) into a mesoporous nanostructure is a molecular transport and lattice rearrangement phenomenon, and must be studied at this length scale (angstrom/nano-scale). In situ Environmental (E)TEM studies, which monitor and characterize nanostructures in gas/vapor environments, can provide unprecedented insight into the fundamental molecular transport and structural transformation processes directly related to bulk properties of gasstorage/release.

In this work [4], we have used in situ ETEM characterization (TEM imaging and diffraction) to study a prototypical breathing MOF, MIL-53(Cr), which is known to store-and-release $\mathrm{H}_{2} \mathrm{O}$ molecules when cyclically heated and cooled in a water-vapor environment, but where the details of this breathing process, or the extent of storage-and-release, are not known. By acquiring in situ ETEM diffraction patterns and low-dose images of a single-crystalline MIL-53(Cr) nanoparticle during heating-cooling in water-vapor (Figure 1), and correlating these experimental data with molecular dynamics (MD) simulations of the MIL-53(Cr) lattice at the same environmental conditions, we were able to precisely determine the number of water molecules adsorbed-desorbed during reversible breathing cycles, and identify the mechanism of activation of the structure that occurs during the first heating (calcination) step. The MIL-53(Cr) breathing process (in $\mathrm{H}_{2} \mathrm{O}$-vapor) is initially primed by the removal of $\mathrm{H}_{2}$ bdc (residual solvent from the synthesis) from the MOF's pore channels when lightly irradiated by the $\mathrm{e}^{-}$beam in UH-vacuum (Figure 1a), with no lattice change or gas absorption following the introduction of $\mathrm{H}_{2} \mathrm{O}$ vapor at room temperature (Figure 1b). When the MOF is heated to $300{ }^{\circ} \mathrm{C}$ (Figure 1c), $1 \mathrm{H}_{2} \mathrm{O}$ molecule per unit cell is adsorbed into the pore channels (change in lattice structure and diffraction pattern), and when cooled back to $27{ }^{\circ} \mathrm{C}$ (Figure 1d), an additional $24 \mathrm{H}_{2} \mathrm{O}$ molecules (per unit cell) are adsorbed in the pores (further change in lattice structure and diffraction pattern). This breathing transition between 1 and $25 \mathrm{H}_{2} \mathrm{O}$ molecules is reversible by heating-cooling cycling. Adsorption of a single water molecule per unit cell during the first heating step to $300{ }^{\circ} \mathrm{C}$ activates the breathing effect, transitioning the lattice to accommodate up to $25 \mathrm{H}_{2} \mathrm{O}$ molecules when subsequently cooled and cycled (heating-cooling). The ETEM-MD approach that we have 
employed in this study establishes an experimental method to precisely characterize the gas absorptionrelease in individual nanostructures at the nanoscale, and should serve as the foundation for additional studies of other MOF systems and nanoporous materials using a similar approach (in situ ETEM correlated with MD simulation) [5].

\section{References:}

[1] N. Stock and S. Biswas, Chem. Rev. 112 (2012), p. 933.

[2] J. Zhao et al., Adv. Mater. Interfaces 1 (2014), p. 1400040.

[3] L. Chen et al., J. Am. Chem. Soc. 135 (2013), p. 15763.

[4] L. R. Parent et al., J. Am. Chem. Soc. 139 (2017), p. 13973.

[5] This work was supported by: L.R.P. National Institutes of Health (NIH) F32 fellowship award F32EB021859, Army Research Office award W911NF-15-1-0189, NSF grant ACI-1053575, Department of Energy (DoE) Contract DE-AC02-05CH11231. A portion of this work was performed using the Environmental Molecular Sciences Laboratory (EMSL), located at Pacific Northwest National Laboratory (PNNL), under EMSL user proposal no. 49590. PNNL is operated by Battelle 454 for DOE under contract DE-AC05-76RL01830. A portion of this work was performed at the EPIC facility at Northwestern University's NUANCE Center supported by NSF awards ECCS-1542205 and DMR-1121262, the International Institute for Nanotechnology (IIN), the Keck Foundation; and the State of Illinois.

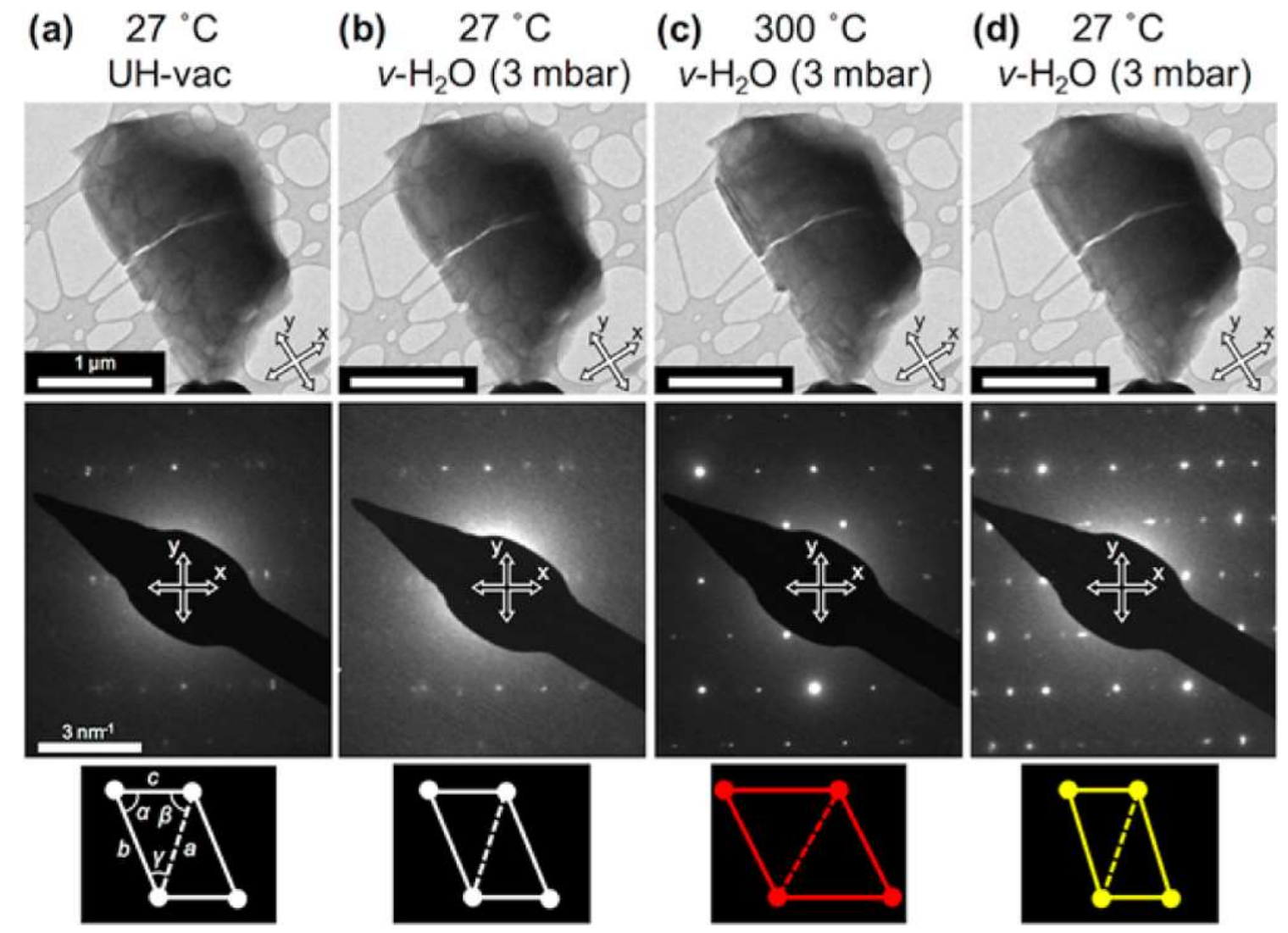

Figure 1. In situ ETEM images (top row) and diffraction patterns (middle row) of one MIL-53(Cr) nanocrystal on the [322] zone axis at four different environmental conditions during breathing; (a) $27{ }^{\circ} \mathrm{C}$ and UH- vacuum, (b) 27 ${ }^{\circ} \mathrm{C}$ and water vapor ("pre-activation"), (c) $300{ }^{\circ} \mathrm{C}$ and water vapor ("calcinated"), and (d) $27{ }^{\circ} \mathrm{C}$ and water vapor ("post-calcination"). The bottom row illustrates the MOF's lattice translons in diffraction space viewed on the [322] zone axis. (Figure reproduced from ref. [4]) 